\title{
Cerebral venous thrombosis without thrombocytopenia after a single dose of COVID-19 (Ad26.COV2.S) vaccine injection: a case report
}

\author{
Martina Di Pietro ${ }^{1} \cdot$ Fedele Dono ${ }^{1,2}(0)$ Stefano Consoli ${ }^{1}$. Giacomo Evangelista ${ }^{1} \cdot$ Valeria Pozzilli $^{1} \cdot$ Dario Calisi $^{1}$. \\ Filomena Barbone $^{3} \cdot$ Laura Bonanni $^{4} \cdot$ Marco Onofrj $^{1} \cdot$ Maria Vittoria De Angelis $^{5} \cdot$ Stefano L. Sensi ${ }^{1,2}$
}

Received: 26 November 2021 / Accepted: 20 February 2022 / Published online: 25 February 2022

(c) Fondazione Società Italiana di Neurologia 2022

\begin{abstract}
Background The coronavirus pandemic became the hard challenge for the modern global health system. To date, vaccination is the best strategy against Sars-Cov-2-related illness. About 3 billions of people received at least one of the approved vaccines. The related adverse events were reported during the various experimental phases, but newer and less common side effects are emerging post-marketing. Vaccine-induced thrombocytopenia with thrombosis (VITT) is one of these insidious adverse reactions and it is considered responsible of venous thrombosis, in both the splanchnic and the cerebral circulation. Although its mechanism has been presumably established, resembling that observed in heparin-induced thrombocytopenia, some venous thromboses seem not to recognize this etiology and their pathogenesis remains unknown. Here we described a case of cerebral venous thrombosis after administration of the Ad26.COV2.S, presenting without thrombocytopenia, paving the way for possible novel causes of this vaccine-induced pathological condition.

Case presentation A 45-year-old woman came to our observation for bilateral periorbital headache associated with retroorbital pain started 8 days after administration of COVID vaccine Jannsen. Ophthalmologic exam showing a bilateral papilledema raised the suspicion of intracranial hypertension. Cerebral magnetic resonance imaging revealed signal alteration with T1-positive contrast enhancement in the right temporal and insular lobes suggestive of cerebral venous thrombosis. The absence of thrombocytopenia and platelet factor 4 (PF-4) antibodies led the clinicians to rule out VITT. The patient was treated successfully with warfarin.

Conclusion Venous thrombosis occurring after COVID-19 vaccination represents an adverse event of special interest. Patients with thrombosis and thrombocytopenia appear to be affected by a general thrombophilic state, sustained by an autoimmune mechanism, and show a higher mortality. Thrombosis without thrombocytopenia's pathogenesis has not yet been clarified, but laboratory data and good response to vitamin $\mathrm{K}$ antagonists help clinicians in the differential diagnosis with VITT. Future research will allow us to discover other possible mechanisms and maybe identify a subgroup of patients with a higher risk of developing this medical complication.
\end{abstract}

Keywords COVID-19 $\cdot$ SARS-CoV-2 infection $\cdot$ Thrombosis $\cdot$ Stroke

\section{Introduction}

Coronavirus disease (COVID-19) due to severe acute respiratory syndrome coronavirus 2 (SARS-CoV-2) infection has affected tens of millions of people [1] globally since it was declared a pandemic by the World Health Organization

Fedele Dono

fedele.dono@unich.it

Stefano L. Sensi ssensi@unich.it

Extended author information available on the last page of the article on March 11, 2020. The development of vaccines started as soon as the virus genome was published in early January 2021 [2]. Several types of COVID-19 vaccines have been produced with different techniques and consequently different mechanisms of action.

Even though the efficacy of the COVID-19 vaccine has been confirmed in the registration trial, some concerns regarding safety issues have been pointed out. One of the most frequently reported COVID-19 vaccines' severe side effects (SAE) is thrombosis. Thromboembolic complications have been observed after administration of two adenovirus vector vaccines, namely ChAdOx $1 \mathrm{nCoV}-19$ 
(Vaxzevria) and Janssen/Johnson \&Johnson COVID19 (Ad26.COV2.S) vaccines. In April 2021, among 34 million people vaccinated with ChAdOx1 nCoV-19 (Vaxzevria), the European Medicine Agency (EMA) has reviewed 163 cases of cerebral venous sinus thrombosis (CVST) and 53 in splanchnic veins, with an estimated incidence of 1:100,000 [3]. Similarly, 17 thromboembolic events were reported over 7.98 million people after Janssen/Johnson\&Johnson COVID-19 (Ad26.COV2.S) vaccination, among which 14 were cases of CVST [4]. COVID-19 vaccine-induced thrombosis is characterized by an atypical localization of the thrombi (e.g., splanchnic and cerebral veins) and frequent association with thrombocytopenia. It has been supposed that Vaccine-induced thrombotic thrombocytopenia (VITT) develops as a consequence of an autoimmune mechanism, presumably due to an immune response to platelet-factor 4 (PF4), similar to heparin-induced thrombocytopenia (HIT). In this context, PF4 binds to polyanions such as heparin and undergoes a conformational change, forming an immunogenic complex that stimulates an anti-PF4-heparin IgG. The resulting immunocomplex causes a strong platelet activation and a decrease in platelet count due to consumption [5]. Since no reports of CVST with thrombocytopenia following authorized mRNA COVID-19 vaccines have been reported, a prothrombotic effect of the adenovirus vector has been supposed [6]. Indeed, it binds to PF4, resambling the action of heparin or an endogenous polyanion, and the resulting immunogenic complex activates the immune system, as described before. However, there is still no unequivocal evidence about the pathophysiology of the thromboembolic events observed after vaccination because a consistent number of these events are not accompanied by thrombocytopenia and PF4 antibodies.

In this report, we describe a case of cerebral venous thrombosis (CVT) following immunization with the Janssen/Johnson \&Johnson COVID-19 (Ad26.COV2.S) vaccine in a woman without any prothrombotic comorbidities. The absence of anti PF4 antibodies and thrombocytopenia and the remarkable response to warfarin therapy, contraindicated in VITT, sheds new light regarding other possible mechanisms of this serious and possibly fatal adverse event.

\section{Case report}

On June 22nd, 2021, a 45-year old woman was admitted to the hospital complaining of a persistent bilateral frontal headache and retro-orbital pain started 8 days before. The patient's past medical history included migraine without aura in prophylactic treatment with beta-blocker (propanolol $20 \mathrm{mg}$ two times/day). No history of previous systemic thrombotic events or spontaneous abortions was reported. During the medical interview, the patient reported being injected with a single dose of viral vector Janssen/Johnson\&Johnson COVID-19 (Ad26.COV2.S) vaccine 2 weeks before the onset of the aforementioned symptoms.

At admission, the patient neurological examination was normal. However, the fundus oculi inspection revealed bilateral papilledema. Hence, the patient underwent a brain computed tomography (CT) with venous and arterial angiography sequences of intracranial and extracranial

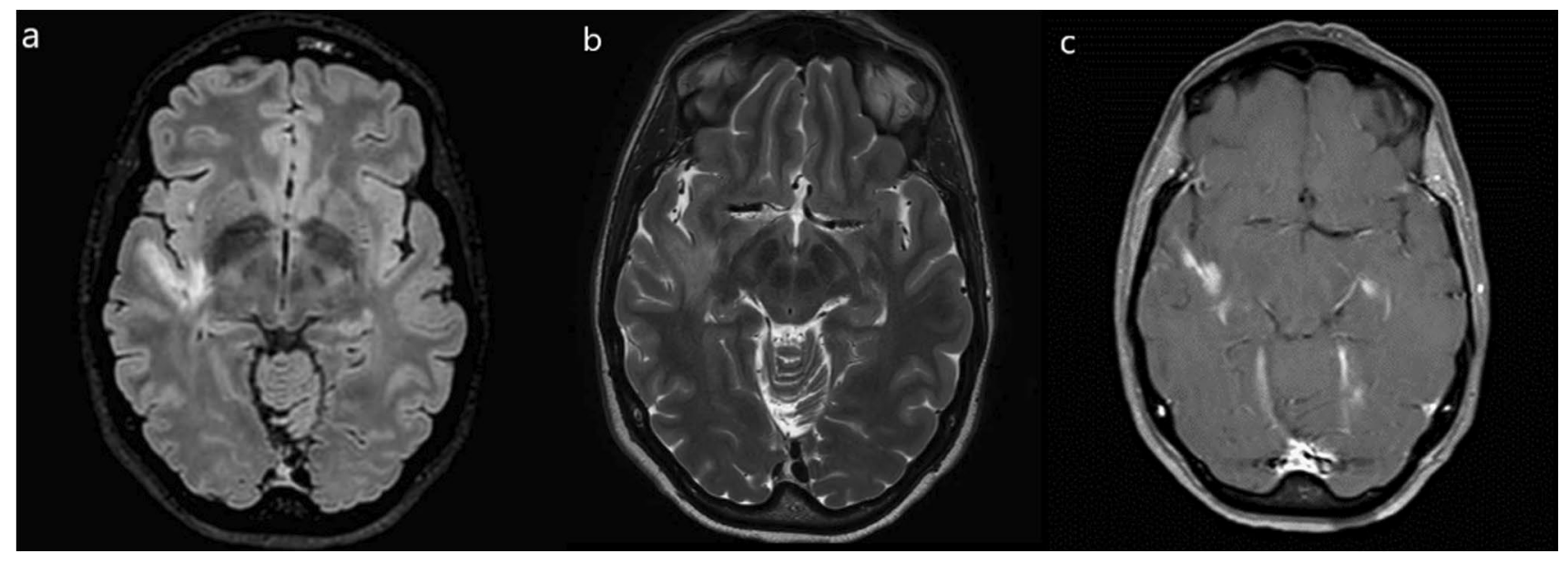

Fig. 1 Cerebral MRI performed at admission with signs of cerebral venous thrombosis. Fluid attenuated inversion recovery sequence (FLAIR) (a) and T2 (b) axial MRI showing hyperintense signal in right temporal and insular subcortical white matter, with "patchy" aspect and no mass effect. Axial T1 post-gadolinium (c) showing high contrast enhancement. These findings are suggestive for venous infarction 
vessels, which showed a blurred hyperdense signal alteration in the cortical-subcortical portion of the right temporal lobe. Thus, a brain magnetic resonance image (MRI) was performed which revealed a hyperintense signal in the right temporal and insular lobes in the T2-Fluid attenuated inversion recovery (FLAIR) sequences with a T1-positive contrast enhancement suggestive of cerebral venous thrombosis in association with brain oedema (Fig. 1). Considering the recent vaccination, a case of VITT was suspected and anticoagulation therapy with Fondaparinux $(7.5 \mathrm{mg} /$ day) was started. Then, blood examination tests were performed which included coagulation assessment, thrombophilia tests, and auto-antibodies screening, which resulted all within the normal range (Table 1). Anti-platelet factor 4 autoantibodies (anti-PF4) were absent, excluding the diagnosis of VITT. Thus, according to the AHA/ASA guidelines for cerebral venous thrombosis ${ }^{9}$, the patient was gradually shifted to warfarin. Furthermore, corticosteroids (dexamethasone ev $12 \mathrm{mg} /$ day) and acetazolamide $(500 \mathrm{mg} /$ day $)$ were started to reduce cerebral edema and intracranial pressure.

In the following days, the clinical condition gradually improved with complete regression of the headache and the papilledema. Two follow-up brain MRI, performed at day 26 and at day 60 after the symptoms' onset, showed a progressive reduction of the signal alterations and the disappearance of the T1 contrast enhancement and the signs of brain oedema (Fig. 2).

\section{Discussion}

CVST has an annual incidence of 3 to 4 cases per million, with a significant female predominance in young adults (aged 31-50) who have inherited or acquired prothrombotic risk factors [7]. Post-marketing safety monitoring data for COVID-19 vaccines have reported several cases of CVST [8] with a median time of 8 days between vaccination and symptom onset. In these cases, the association of CVST with thrombocytopenia has been frequently observed, even though several reports of vaccine-induced CVST without thrombocytopenia have been also described [9]. Updated observed-to-expected analysis for CVST after ChAdOx1 nCoV-19 (Vaxzevria) and Janssen/Johnson \&Johnson COVID-19 (Ad26.COV2.S) immunization has shown greater mortality in patients with thrombocytopenia when compared to patients without thrombocytopenia. Thus, low platelet count could be an important risk factor for a worse outcome.

In our report, we describe a case of Janssen/ Johnson\&Johnson COVID-19 (Ad26.COV2.S)-induced CVST without thrombocytopenia. Even though several cases of CVST without thrombocytopenia have been described in patients immunized with ChAdOx $1 \mathrm{nCoV}-19$ (Vaxzevria), no reports are available so far on this possible SAE from Janssen/Johnson\&Johnson COVID-19 (Ad26. COV2.S) vaccination. The mechanism through which COVID-19 vaccine might exert a prothrombotic effect without inducing thrombocytopenia is largely unknown, but there is strong evidence for the interaction between SarsCov2 virus and angiotensin-converting enzyme 2 (ACE2) receptor as a possible cause of platelet aggregation. Considering the mechanism through which viral vector vaccines induced immunization (simulation of S glycoprotein synthesis by the organism), binding of the $\mathrm{S}$ glycoprotein to ACE2 may lead to ACE2 downregulation, which in turn

Table 1 Main laboratory data in patients affected by CVT

\begin{tabular}{|c|c|c|}
\hline & Values & Laboratory normal ranges \\
\hline $\mathrm{Hb}$ & $15.0 \mathrm{~g} / \mathrm{dl}$ & $12-16 \mathrm{~g} / \mathrm{dl}$ \\
\hline PLT & $329,000 \mathrm{mmc}$ & $150,000-450,000 \mathrm{mmc}$ \\
\hline PT & $106.8 \%$ & $80-120 \%$ \\
\hline aPTT & $28 \mathrm{~s}$ & $26-37 \mathrm{~s}$ \\
\hline INR & 1.04 & $0.95-1.15$ \\
\hline D-dimer & $0.26 \mathrm{mg} / \mathrm{l}$ & $0-0.50 \mathrm{mg} / \mathrm{l}$ \\
\hline Anti PF4 antibody & Negative & \\
\hline AT III & $115 \%$ & $83-128 \%$ \\
\hline Protein $\mathrm{C}$ & $121 \%$ & $60-140 \%$ \\
\hline Protein S & $77 \%$ & $55-160 \%$ \\
\hline $\begin{array}{l}\text { Activated protein } \mathrm{C} \text { resist- } \\
\text { ance }\end{array}$ & 3.40 ratio & $>2.20$ ratio \\
\hline Factor V Leiden & No mutation & \\
\hline Prothrombin G20210 & No mutation & \\
\hline ANA & Absent & \\
\hline AMA & Absent & \\
\hline SMAs & Absent & \\
\hline c-ANCA & $0.0 \mathrm{UA} / \mathrm{ml}$ & $<20 \mathrm{UA} / \mathrm{ml}$ \\
\hline p-ANCA & $0.2 \mathrm{UA} / \mathrm{ml}$ & $<20 \mathrm{UA} / \mathrm{ml}$ \\
\hline $\begin{array}{l}\text { Anti-beta-2 glycoprotein } \\
\text { IgG }\end{array}$ & $1.0 \mathrm{UA} / \mathrm{ml}$ & $<20 \mathrm{UA} / \mathrm{ml}$ \\
\hline $\begin{array}{l}\text { Ab-beta-2 glycoprotein } \\
\text { IgM }\end{array}$ & $0.3 \mathrm{UA} / \mathrm{ml}$ & $<10 \mathrm{UA} / \mathrm{ml}$ \\
\hline Antiphospholipid IgG & $0.5 \mathrm{GPL} / \mathrm{ml}$ & $<10 \mathrm{GPL} / \mathrm{ml}$ \\
\hline Antiphospholipid IgM & $0.9 \mathrm{MLP} / \mathrm{ml}$ & $<10 \mathrm{MLP} / \mathrm{ml}$ \\
\hline Anticardiolipin IgG & $0.4 \mathrm{GPL} / \mathrm{ml}$ & $<20 \mathrm{GPL} / \mathrm{ml}$ \\
\hline Anticardiolipin IgM & $0.0 \mathrm{MLP} / \mathrm{ml}$ & $<10 \mathrm{MLP} / \mathrm{ml}$ \\
\hline ESR & $6 \mathrm{~mm}$ & $2-15 \mathrm{~mm}$ \\
\hline PCR & $0.33 \mathrm{mg} / 1$ & $0-5 \mathrm{mg} / \mathrm{l}$ \\
\hline
\end{tabular}

Abbreviations: $H b$, hemoglobin; $P L T$, platelet count; $P T$, prothrombin time; $a P T T$, activated partial thromboplastin time; $I N R$, international normalized ratio; anti $P F-4$, anti-platelet factor 4; $A T$, antithrombin; $A N A$, antinuclear antibodies; $A M A$, anti-mitocondrial antibodies; $S M A s$, smooth muscle antibodies; ANCA, anti-neutrophilic cytoplasmic antibodies; $E S R$, erythrocyte sedimentation rate; $P C R$, reactive $\mathrm{C}$ protein 
Fig. 2 Cerebral MRI performed after anticoagulant therapy which shows progressive resolution of cerebral venous thrombosis. MRI images at 26 days after symptoms onset showing the clear reduction of right temporal signal alteration in FLAIR (a), involving only the right temporal gyrus, and significant reduction of contrast enhancement (b), visible in the underlying venous. At 60 days, further reduction of temporal hyperintensity (c) and complete resolution of contrast enhancement (d)

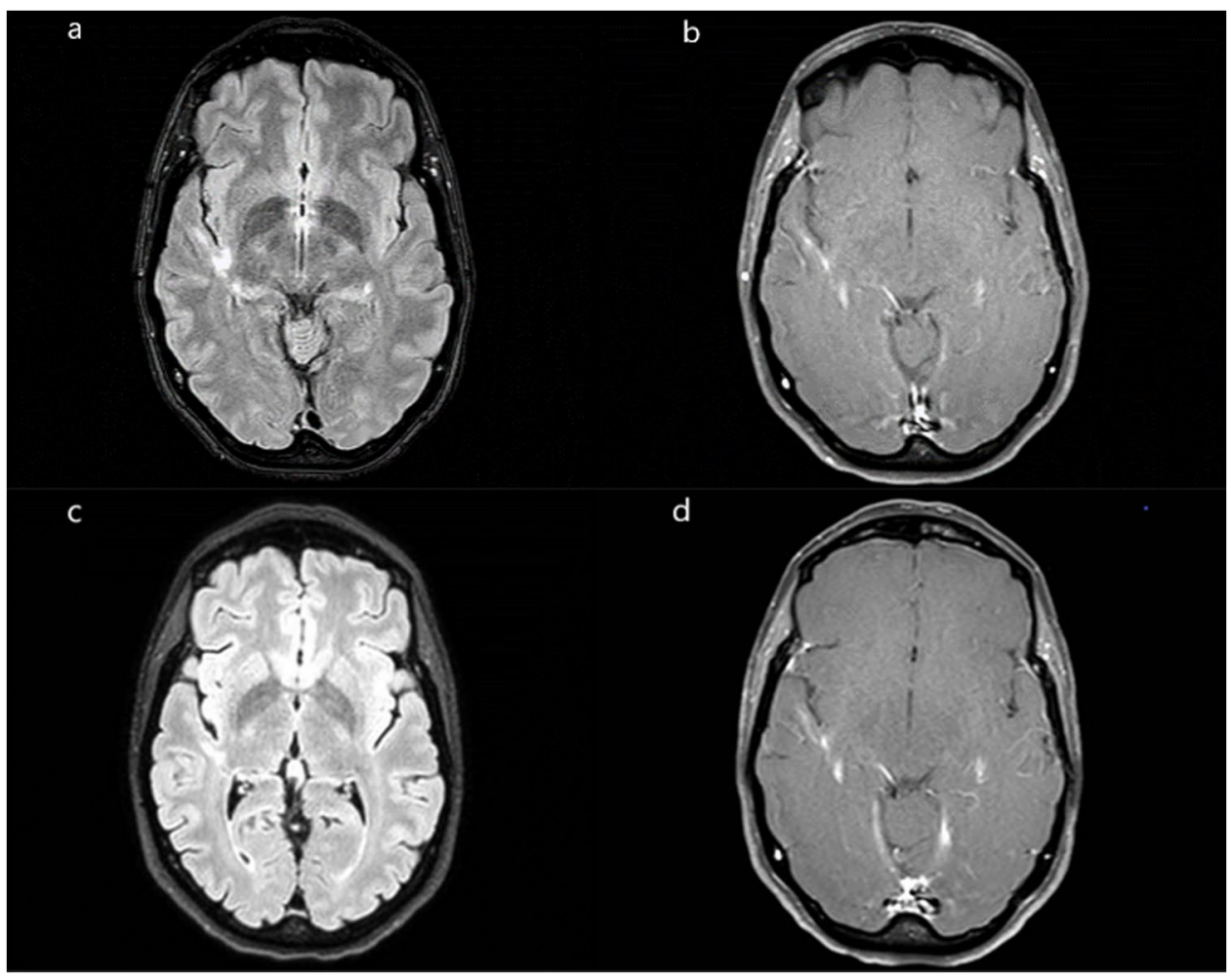

results in a higher formation of Angiotensin II (Ang II) by ACE, and less conversion of ACE2 to Ang-(1-7). The overactivation of ACE/AngII/AT1R pathway, with its prothrombotic, pro-inflammatory, and vasoconstrictor effect, could be responsible of stroke and thrombosis, also in the venous circulation [10]. Thus, it can be speculated that in a selected group of subjects, this vaccination may elicit a strong inflammatory response leading to platelet aggregation and clot formation. However, in our report, no evidence of pro-inflammatory response to vaccination has been evidenced according to blood examination, hence rejecting the possible role of an inflammatory-mediated mechanism. Undoubtfully, in about 35-40\% of CVST a predisposing factor is not always identified [11] and vaccines can be considered themselves a pro-thrombotic trigger. On the other hand, it has to be reminded that CVST may happen in general population independently of vaccination, where it is often characterized by a normal platelet count. In our case, the lack of any previous prothrombotic state and the temporal association between vaccine administration and the onset of the clinical picture support the hypothesis of a causal role of the Janssen/Johnson\&Johnson COVID-19 (Ad26.COV2.S) vaccine in the genesis of CVST, although not unequivocally proven.

Therapeutic management of vaccine-induced CVST without thrombocytopenia is different from the treatment of VITT. In fact, in VITT, anticoagulation therapy with low molecular weight heparin (LMWH) and vitamin $\mathrm{K}$-antagonists is contraindicated due to the risk of worsening of thrombosis. Novel oral anticoagulants (NOACs) instead (dabigatran, apixaban, edoxaban, and rivaroxaban) and fondaparinux can be safely used. Urgent administration of high-dose intravenous immunoglobulin $(0.5-1 \mathrm{~g} / \mathrm{kg}$ for 2 days) is also recommended [12]. On the contrary, in the management of CVST without thrombocytopenia, heparins can be used and are associated with a more favorable outcome [13].

\section{Conclusion}

Thrombotic events following COVID-19 vaccination have a higher incidence in those patients who receive a viral vector vaccine, such as Vaxzevria and Jannsen, especially when associated to thrombocytopenia. However, vaccinerelated CVST without thrombocytopenia has also been described. According to our experience and in line with the current guidelines, normal platelet count and the absence of anti-PF4 antibodies ruled out the diagnosis of VITT and authorized us to treat vaccine-related CVT likewise as sporadic CVST. Further studies are needed to clarify the specific etiologies of this condition, to identify subgroups of patients with an increased risk of developing CVST and avoid this complication. 
Author contribution MDP and FD contributed to the conception and design of the study. MDP and FD wrote the manuscript. All authors contributed to manuscript revisions, and read and approved the submitted version.

\section{Declarations}

Ethical approval The paper is exempt from ethical committee approval because it is a case report.

Informed consent Written informed consent was obtained from the patient for publication of this case report and any accompanying images.

Conflict of interest The authors declare no competing interests.

\section{References}

1. Chen Y, Cheng L, Lian R, Song Z, Tian J (2021) COVID-19 vaccine research focusses on safety, efficacy, immunoinformatics, and vaccine production and delivery: a bibliometric analysis based on VOSviewer. Biosci Trends 15(2):64-73. https://doi.org/10.5582/ bst.2021.01061

2. Dos Santos WG. Impact of virus genetic variability and host immunity for the success of COVID-19 vaccines. Biomed Pharmacother. 2021 Apr;136:111272. https://doi.org/10.1016/j.biopha. 2021.111272.

3. Tran Kiem C, Andronico A, Bosetti P, Paireau J, Alter L, Boëlle PY, Fontanet A, Lévy-Bruhl D, Cauchemez S (2021) Benefits and risks associated with different uses of the COVID-19 vaccine Vaxzevria: a modelling study, France, May to September 2021. Euro Surveill 26(26):2100533. https://doi.org/10.2807/1560-7917

4. Shay DK, Gee J, Su JR, Myers TR, Marquez P, Liu R, Zhang B, Licata C, Clark TA, Shimabukuro TT (2021) Safety monitoring of the Janssen (Johnson \& Johnson) COVID-19 vaccine - United States, March-April 2021. MMWR Morb Mortal Wkly Rep 70(18):680-684. https://doi.org/10.15585/mmwr.mm7018e2

5. Greinacher A (2015) Heparin-induced thrombocytopenia. N Engl J Med 373(19):1883-1884. https://doi.org/10.1056/NEJMc1510993

6. Wolins N, Lozier J, Eggerman TL, Jones E, Aguilar-Córdova E, Vostal JG (2003) Intravenous administration of replication-incompetent adenovirus to rhesus monkeys induces thrombocytopenia by increasing in vivo platelet clearance. Br J Haematol 123(5):903905. https://doi.org/10.1046/j.1365-2141.2003.04719.x (PMID: 14632782)

7. Tadi P, Behgam B, Baruffi S. Cerebral Venous Thrombosis. 2021 Aug 11. In: StatPearls [Internet]. Treasure Island (FL): StatPearls Publishing; 2021 Jan-. PMID: 29083599.

8. See I, Su JR, Lale A, Woo EJ, Guh AY, Shimabukuro TT, Streiff MB, Rao AK, Wheeler AP, Beavers SF, Durbin AP, Edwards K, Miller E, Harrington TA, Mba-Jonas A, Nair N, Nguyen DT, Talaat KR, Urrutia VC, Walker SC, Creech CB, Clark TA, DeStefano F, Broder KR (2021) US case reports of cerebral venous sinus thrombosis with thrombocytopenia after Ad26.COV2.S vaccination, March 2 to April 21, 2021. JAMA 325(24):2448-2456. https://doi.org/10.1001/jama.2021.7517.

9. Dutta A, Ghosh R, Bhattacharya D, Bhat S, Ray A, Pandit A, Das S, Dubey S (2021) Anti-PF4 antibody negative cerebral venous sinus thrombosis without thrombocytopenia following immunization with COVID-19 vaccine in an elderly non-comorbid Indian male, managed with conventional heparin-warfarin based anticoagulation. Diabetes Metab Syndr 15(4):102184. https://doi.org/ 10.1016/j.dsx.2021.06.021

10. Divani AA, Andalib S, DiNapoli M, Lattanzi S, Hussain MS, Biller J, McCullough LD, Azarpazhooh MR, Seletska A, Mayer SA, Torbey M (2020) Coronavirus disease 2019 and stroke: clinical manifestations and pathophysiological insights. J Stroke Cerebrovasc Dis 29(8):104941. https://doi.org/10.1016/j.jstrokecer ebrovasdis.2020.104941

11. Alvis-Miranda HR, Milena Castellar-Leones S, Alcala-Cerra G, Rafael M-S (2013) Cerebral sinus venous thrombosis. J Neurosci Rural Pract 4(4):427-438. https://doi.org/10.4103/0976-3147. 120236

12. Tsilingiris D, Vallianou NG, Karampela I, Dalamaga M (2021) Vaccine induced thrombotic thrombocytopenia: the shady chapter of a success story. Metabol Open 11:100101. https://doi.org/10. 1016/j.metop.2021.100101

13. Filippidis A, Kapsalaki E, Patramani G, Fountas KN (2009) Cerebral venous sinus thrombosis: review of the demographics, pathophysiology, current diagnosis, and treatment. Neurosurg Focus 27(5):E3. https://doi.org/10.3171/2009.8.FOCUS09167

Publisher's note Springer Nature remains neutral with regard to jurisdictional claims in published maps and institutional affiliations. 


\section{Authors and Affiliations}

Martina Di Pietro ${ }^{1} \cdot$ Fedele Dono $^{1,2}(1)$. Stefano Consoli ${ }^{1}$. Giacomo Evangelista ${ }^{1} \cdot$ Valeria Pozzilli $^{1}$ - Dario Calisi ${ }^{1}$. Filomena Barbone ${ }^{3} \cdot$ Laura Bonanni $^{4} \cdot$ Marco Onofrj $^{1} \cdot$ Maria Vittoria De Angelis $^{5}$. Stefano L. Sensi ${ }^{1,2}$

1 Department of Neuroscience, Imaging, and Clinical Sciences, "G. D'Annunzio" University of Chieti-Pescara, Chieti, Italy

2 Behavioral Neurology and Molecular Neurology Units, Center for Advanced Studies and Technology - CAST-, "G. D’Annunzio" University of Chieti-Pescara, Chieti, Italy

3 Department of Neurology, "SS Annunziata" Hospital, Chieti, Italy
4 Department of Medicine and Aging Sciences, "G. D’Annunzio" University of Chieti-Pescara, Chieti, Italy

5 Department of Neurology, Stroke Unit, "SS Annunziata" Hospital, Chieti, Italy 